\title{
Electromagnetic Waves Along an Infinitely Long and Thin Conducting Wire in a Magneto- Ionic Medium
}

\author{
Yasuto Mushiake \\ Faculty of Engineering, Tohoku University, Sendai, Japan
}

(Received October 14, 1964; revised November 24, 1964)

\begin{abstract}
Electromagnetic fields of an infinitely long conducting cylinder in a magneto-ionic medium with axial static magnetic field are theoretically analyzed. Expressions of the electromagnetic fields and the dispersion formulas are obtained. Explicit approximate expressions of the relative propagation constants for the case of an extremely thin wire are derived and their dependence on the plasma and the cyclotron frequencies is discussed. The behavior of the electromagnetic fields around the wire is also discussed.
\end{abstract}

\section{Introduction}

Extensive investigations have already been made of the electromagnetic wave propagation in an anisotropic medium. However, the characteristics of antennas placed in such a medium [Kogelnik, 1960; Marini, 1963; Wu, 1963] have not been thoroughly studied. For example, the wavelength of the current distribution along an antenna wire has not been obtained, while the radiation impedances of linear antennas have already been computed with assumed current distributions [Ament et al., 1964].

The purpose of this paper is to present some results obtained by a theoretical analysis of the electromagnetic waves along an infinitely long and thin conducting wire in a magnetoionic medium with the axial static magnetic field [Mushiake, 1964]

In this theory the electric property of the magneto-ionic medium is assumed to be expressed by the well-known tensor dielectric constant, and the existence of ion sheath is neglected.

Expressions of electromagnetic fields and the dispersion formulas are obtained. Explicit approximate expressions of the relative propagation constants for the case of an extremely thin wire are derived and their dependence on the plasma frequency and the cyclotron frequency is studied. The behavior of the electromagnetic fields of the extremely thin wire will also be discussed.

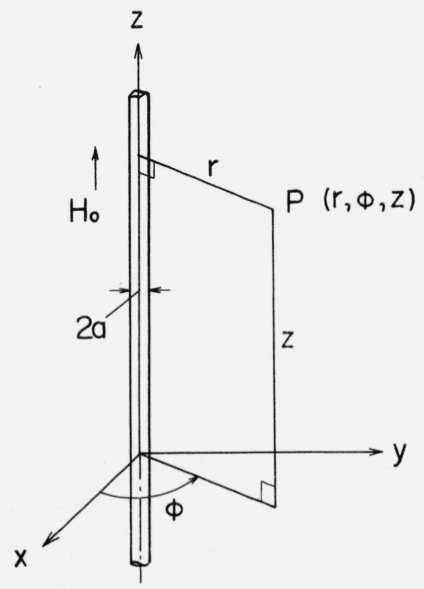

FIGURE 1. Geometry of a circular cylinder. 


\section{Formulation for the Electromagnetic Fields}

The geometry of an infinitely long circular cylinder of perfect conductivity is shown in figure 1. From Maxwell's equation in a lossless magneto-ionic medium, the following equation for the electric field $E$ can be derived:

where

$$
\nabla \times \nabla \times E=k_{0}^{2}(\kappa) \cdot E
$$

$$
\begin{aligned}
(\kappa)=\left(\begin{array}{lll}
\kappa_{1} & j \kappa_{2} & 0 \\
-j \kappa_{2} & \kappa_{1} & 0 \\
0 & 0 & \kappa_{3}
\end{array}\right) & =\left(\begin{array}{ccc}
1-\frac{X}{1-Y^{2}} & -j \frac{X Y}{1-Y^{2}} & 0 \\
j \frac{X Y}{1-Y^{2}} & 1-\frac{X}{1-Y^{2}} & 0 \\
0 & 0 & 1-X
\end{array}\right), \\
X & =\omega_{p}^{2} / \omega^{2} \\
Y & =\omega_{H} / \omega \\
k_{0}^{2} & =\omega^{2} \mu_{0} \epsilon_{0},
\end{aligned}
$$

and $\omega, \omega_{p}$, and $\omega_{H}$ are the angular wave frequency, the angular electron plasma frequency, and the angular electron gyrofrequency, respectively. The time factor of $e^{j \omega t}$ is assumed, and MKS units are used in the present paper.

The axially symmetric solution of (1) for the electric field can be written as

$$
\begin{aligned}
& E_{r}=E_{0 r} H_{1}^{(2)}\left(k_{r} r\right) e^{-j k_{z} Z}, \\
& E_{\phi}=E_{0 \phi} H_{1}^{(2)}\left(k_{r} r\right) e^{-j k_{z} Z}, \\
& E_{z}=E_{0 z} H_{0}^{(2)}\left(k_{r} r\right) e^{-j k_{z} Z},
\end{aligned}
$$

where $k_{r}$ and $k_{z}$ are the propagation constants, and the amplitude factors $E_{0 r}, E_{0 \phi}$, and $E_{0 z}$ must satisfy three relations:

$$
\begin{gathered}
\left(\kappa_{1}+\frac{n_{z}^{2} \kappa_{3}}{n_{r}^{2}-\kappa_{3}}\right) E_{0 r}+j \kappa_{2} E_{0 \phi}=0, \\
-j \kappa_{2} E_{0 r}+\left(\kappa_{1}-n_{r}^{2}-n_{z}^{2}\right) E_{0 \phi}=0, \\
j n_{z} n_{r} E_{0 r}-\left(n_{r}^{2}-\kappa_{3}\right) E_{0 z}=0 .
\end{gathered}
$$

In these equations $n_{r}$ and $n_{z}$ are the relative propagation constants defined by $n_{r}=k_{r} / k_{0}$ and $n_{z}=k_{z} / k_{0}$, and they have complex values of negative imaginary parts in general. Elimination of the amplitude factors from (7) and (8) leads to

$$
\kappa_{1} n_{r}^{4}+\left(\kappa_{1}+\kappa_{3}\right) n_{r}^{2} n_{z}^{2}-\left(\kappa_{1}^{2}+\kappa_{1} \kappa_{3}-\kappa_{2}^{2}\right) n_{r}^{2}-2 \kappa_{1} \kappa_{3} n_{z}^{2}+\kappa_{3} n_{z}^{4}+\left(\kappa_{1}^{2}-\kappa_{2}^{2}\right) \kappa_{3}=0
$$

This equation gives a relation between the relative propagation constants $n_{r}$ and $n_{z}$.

It is found from (10) that two values of $n_{r}^{2}$ will be determined for one value of $n_{z}^{2}$. In other words, two different wave modes which propagate in the same direction of $+Z$ or $-Z$ along the cylinder with one common wavelength and which have different propagation constants in $r$-direction can exist. The values of various parameters for these two modes will be discriminated by the subscripts 1 and 2 hereafter. By taking two such modes, a general solution of (1) is given by 


$$
\begin{aligned}
& E_{r}=\left\{E_{0 r 1} H_{1}^{(2)}\left(k_{r 1} r\right)+E_{0 r 2} H_{1}^{(2)}\left(k_{r 2} r\right)\right\} e^{\mp j k} z^{Z}, \\
& E_{\phi}=\left\{E_{0 \phi 1} H_{1}^{(2)}\left(k_{r 1} r\right)+E_{0 \phi 2} H_{1}^{(2)}\left(k_{r 2} r\right)\right\} e^{\mp j k} z^{Z}, \\
& E_{z}=\left\{E_{0 z 1} H_{0}^{(2)}\left(k_{r 1} r\right)+E_{0 z 2} H_{0}^{(2)}\left(k_{r 2} r\right)\right\} e^{\mp j k} z^{Z} .
\end{aligned}
$$

Introduction of the boundary conditions $E_{\phi}=0$ and $E_{z}=0$ into (11) at the surface of the cylinder, or $r=a$, yields two relations between amplitude factors for two modes. Eliminating the amplitude factors from these two relations, we obtain

$$
\frac{n_{r 1}\left(\kappa_{1}-n_{z}^{2}-n_{r 1}^{2}\right)}{\kappa_{3}-n_{r 1}^{2}} \cdot \frac{H_{0}^{(2)}\left(k_{0} n_{r 1} a\right)}{H_{1}^{(2)}\left(k_{0} n_{r 1} a\right)}=\frac{n_{r 2}\left(\kappa_{1}-n_{z}^{2}-n_{r 2}^{2}\right)}{\kappa_{3}-n_{r 2}^{2}} \cdot \frac{H_{0}^{(2)}\left(k_{0} n_{r 2} a\right)}{H_{1}^{(2)}\left(k_{0} n_{r 2} a\right)} .
$$

This is another relation for $n_{r 1}, n_{r 2}$, and $n_{z}$. Therefore, the values of these relative propagation constants are definitely prescribed by two simultaneous equations of (10) and (12). This means that these equations are the dispersion relation expressed in the form of simultaneous equations.

Explicit but formal expressions of the electromagnetic fields for this case can easily be obtained after some straightforward calculations as follows:

$$
\left.\begin{array}{l}
E_{r}=E_{0}\left\{H_{1}^{(2)}\left(k_{r 1} r\right)-\frac{\kappa_{1}-n_{z}^{2}-n_{r 2}^{2}}{\kappa_{1}-n_{z}^{2}-n_{r 1}^{2}} \cdot \frac{H_{1}^{(2)}\left(k_{r 1} a\right)}{H_{1}^{(2)}\left(k_{r 2} a\right)} H_{1}^{(2)}\left(k_{r 2} r\right)\right\} e^{\mp j k_{z} Z}, \\
E_{\phi}=E_{0} \frac{j \kappa_{2}}{\kappa_{1}-n_{z}^{2}-n_{r 1}^{2}}\left\{H_{1}^{(2)}\left(k_{r 1} r\right)-\frac{H_{1}^{(2)}\left(k_{r 1} a\right)}{H_{1}^{(2)}\left(k_{r 2} a\right)} H_{1}^{(2)}\left(k_{r 2} r\right)\right\} e^{\mp j k_{z} Z}, \\
E_{z}=-E_{0} \frac{j n_{r 1} n_{z}}{\kappa_{3}-n_{r 1}^{2}}\left\{H_{0}^{(2)}\left(k_{r 1} r\right)-\frac{H_{0}^{(2)}\left(k_{r 1} a\right)}{H_{0}^{(2)}\left(k_{r 2} a\right)} H_{0}^{(2)}\left(k_{r 2} r\right)\right\} e^{\mp j k_{z} Z},
\end{array}\right\}
$$

where an amplitude factor $E_{0}$ is to be determined from the total axial current $I(z)$ on the cylinder by the relation

$$
I(z)=2 \pi a\left[H_{\phi}(z)\right]_{r=a} .
$$

\section{Approximations for Extremely Thin Wire}

It seems that the determination of the values of the relative propagation constants from the simultaneous equations (10) and (12) is not simple in general. For this reason, only the case of an extremely thin wire will be discussed under the assumption of the relations

$$
\left.\begin{array}{l}
\left|k_{r 1} a\right| \ll 1, \quad\left|k_{r 2} a\right| \ll 1, \\
\left|\ln \frac{n_{r 1}}{n_{r 2}}\right| \ll\left|\ln k_{r 1} a\right| .
\end{array}\right\}
$$


Under these conditions (12), which is obtained from the boundary conditions, can be approximated (see appendix) by

$$
\frac{n_{r 1}^{2}\left(\kappa_{1}-n_{z}^{2}-n_{r 1}^{2}\right)}{\kappa_{3}-n_{r 1}^{2}} \simeq \frac{n_{r 2}^{2}\left(\kappa_{1}-n_{z}^{2}-n_{r 2}^{2}\right)}{\kappa_{3}-n_{r 2}^{2}} .
$$

If this approximate equation is used instead of the exact equation (12), the simultaneous equations for the relative propagation constants can be solved analytically, and their values are given explicitly by

$$
\begin{aligned}
& n_{z}^{2} \simeq \kappa_{1}=1-\frac{X}{1-Y^{2}}, \\
& \left.\begin{array}{c}
n_{r 1}^{2} \\
n_{r 2}^{2}
\end{array}\right\} \simeq \frac{\kappa_{2}}{2 \kappa_{1}}\left\{-\kappa_{1} \pm \sqrt{\kappa_{2}^{2}+4 \kappa_{1} \kappa_{3}}\right\} \\
& =\frac{X Y}{2\left(1-X-Y^{2}\right)\left(1-Y^{2}\right)}\left\{-X Y \pm \sqrt{X^{2} Y^{2}+4(1-X)\left(1-Y^{2}\right)\left(1-X-Y^{2}\right)} .\right)
\end{aligned}
$$

Numerical computations have been made of the relative propagation constants with these formulas and some examples of the results are shown in figures 2 and 3 . The values of $n_{z}^{2}$ are also shown on the plane of $X-Y^{2}$ as in figure 4. According to (18), the value of $n_{z}$ is independent of the component of the dielectric tensor parallel to the wire, but it obviously depends upon the values of $X$ and $Y$.

As these results are obtained under the assumption of (16), those assumed conditions must be examined. That will be done in the next section.

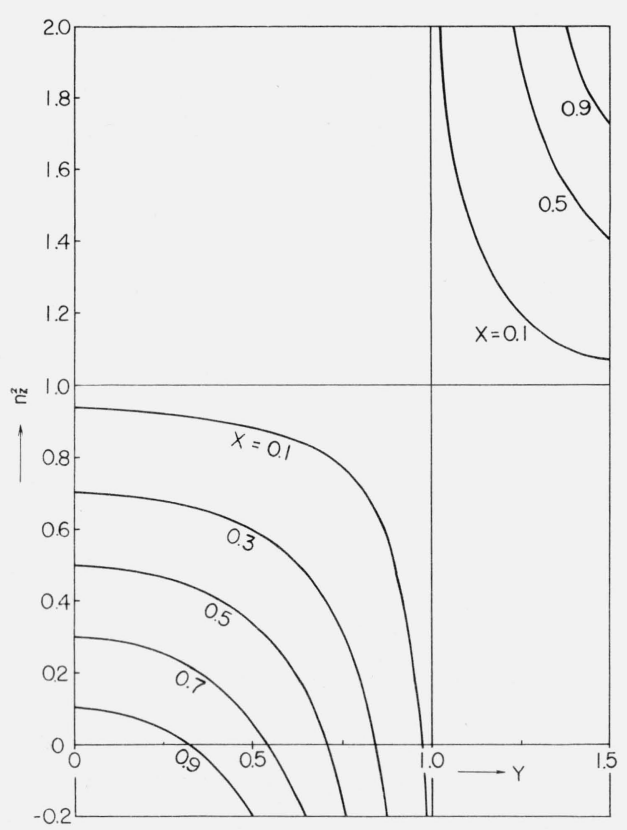

FIGURE 2. Influence of the static magnetic field on the values of the relative propagation constant $n_{z}\left(n_{z}^{2}\right.$ versus $\mathrm{Y}$ with parameter $\mathrm{X})$.

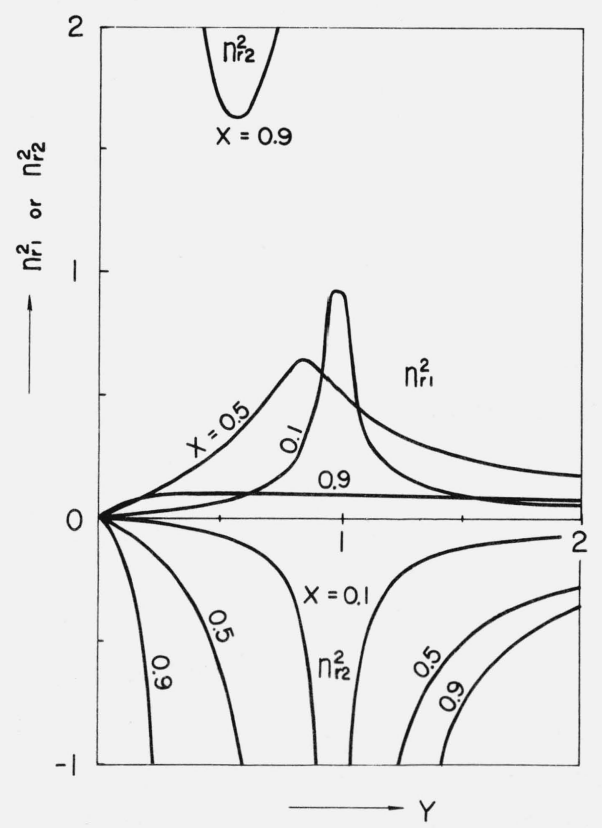

FIGURE 3. Influence of the static magnetic field on the values of the relative propagation constants $\mathrm{n}_{\mathrm{r} 1}$ and $\mathrm{n}_{\mathrm{r} 2}\left(\mathrm{n}_{\mathrm{r} 1}^{2}\right.$ and $\mathrm{n}_{\mathrm{r} 2}^{2}$ versus $\mathrm{Y}$ with parameter $\left.\mathrm{X}\right)$. 


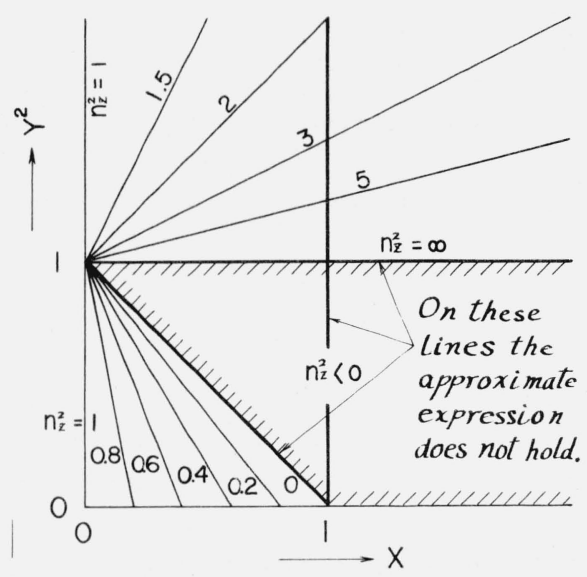

FIGURE 4. Lines for $\mathrm{n}_{\mathrm{z}}^{2}=$ constant on the plane of $\mathrm{X}-\mathrm{Y}^{2}$

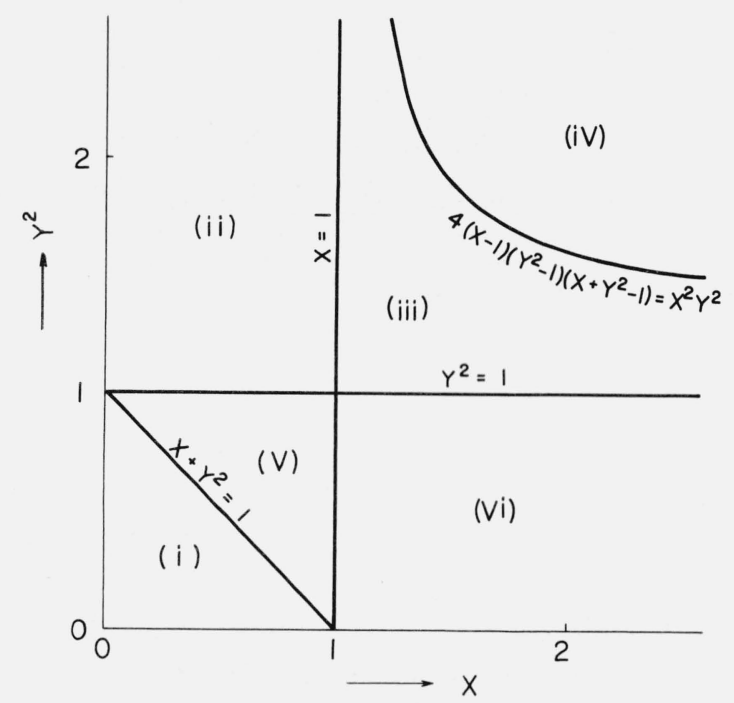

Figure 5. Six regions of $\mathrm{X}-\mathrm{Y}^{2}$ plane.

\section{Discussion of the Relative Propagation Constants}

It is obvious that the assumed relations (16) are not true when either one of $\left|n_{r 1}\right| \gg 1,\left|n_{r 2}\right| \gg 1$, $\left|n_{r 1} / n_{r 2}\right| \simeq 0$ or $\infty$ holds. For convenience in examining these conditions the $X-Y^{2}$ plane is divided into six regions as shown in figure 5 , where the boundaries of the regions are

$$
\left.\begin{array}{ll}
\text { (a) } & X=1, \\
\text { (b) } & Y^{2}=1, \\
\text { (c) } & X+Y^{2}=1, \\
\text { (d) } & 4(X-1)\left(Y^{2}-1\right)\left(X+Y^{2}-1\right)=X^{2} Y^{2} .
\end{array}\right\}
$$

The approximate values of $n_{r 1}^{2}$ and $n_{r 2}^{2}$ in the six regions and on the boundaries are examined from the expressions of (18), and the results are listed below, where the value of $n_{z}^{2}$ is also shown.

$$
\begin{aligned}
& \text { (i) } \quad 0<n_{r 1}^{2}<1-X, \quad n_{r 2}^{2}<0, \quad 0<n_{z}^{2}<1 \text {, } \\
& \text { (ii) } 0<n_{r 1}^{2}<1-X, \quad n_{r 2}^{2}<0, \quad 1<n_{z}^{2} \text {, } \\
& \text { (iii) } \quad n_{r 1}^{2}<0, \quad n_{r 2}^{2}<0, \quad 1<n_{z}^{2} \text {, } \\
& \text { (iv) } n_{r 1}^{2}, n_{r 2}^{2} \text { : complex conjugates, } 1<n_{z}^{2} \text {, } \\
& \text { (v) } \quad 1-X<n_{r 1}^{2}<1, \quad 0<n_{r 2}^{2}, \quad n_{z}^{2}<0 \text {, } \\
& \text { (vi) } \quad n_{r 1}^{2}<0, \quad 0<n_{r 2}^{2}, \quad n_{z}^{2}<0 \text {, }
\end{aligned}
$$




$$
\begin{aligned}
& (X=1) \quad n_{r 1}^{2}=0, \quad n_{r 2}^{2}=1 /\left(1-Y^{2}\right), \quad n_{z}^{2} \leqq 0 \text { or } 1 \leqq n_{z}^{2}, \\
& \left(Y^{2}=1\right) \quad \quad n_{r 1}^{2}=1-X, \quad\left|n_{r z}^{2}\right|=\infty, \quad\left|n_{z}^{2}\right|=\infty, \\
& \left(X+Y^{2}=1\right) \quad n_{r 1}^{2}=1-X, \quad\left|n_{r 2}^{2}\right|=\infty, \quad n_{z}^{2}=0, \\
& (d \text {-line }) \quad n_{r 1}^{2}=n_{r 2}^{2}=2(1-X), \quad 1<n_{z}^{2}, \\
& (X=0) \quad n_{r 1}^{2}=-n_{r 2}^{2}=0, \quad n_{z}^{2}=1, \\
& (Y=0) \quad n_{r 1}^{2}=-n_{r 2}^{2}=0, \quad n_{z}^{2} \leqq 1 .
\end{aligned}
$$

It is found from these results that the approximate expressions (18) for the relative propagations constants do not hold on the lines of $X=1, Y^{2}=1, X+Y^{2}=1$, and in the vicinity of these lines. Further examination reveals that the approximation of (18) does not hold for extremely large value of $X$ either.

Aside from these exceptions, the relations (20) and (21) give various information concerning the waves along an extremely thin wire. Some examples are described below.

When $n_{z}$ is real, the wavelength $\lambda$ along the wire is given, in general, by

$$
\lambda=\lambda_{0} / n_{z},
$$

where $\lambda_{0}$ is the free space wavelength. Therefore, it is found that the wavelength along the wire is longer than $\lambda_{0}$ in the region (i), while $\lambda$ is shorter in the regions (ii), (iii), and (iv). On the other hand, pure imaginary values of $n_{z}$ as in the regions (v) and (vi) represent the attenuating waves in the direction of propagation, $+Z$ or $-Z$.

If $n_{r i}^{2}(i=1$ or 2$)$ is positive real, the electromagnetic field components are proportional to Hankel functions. On the contrary, if $n_{r i}^{2}$ is negative real, the arguments of the Hankel functions become pure imaginary and these functions transform into the modified Bessel functions of the first kind. Consequently, the field components vanish very rapidly with increasing $r$. That is true, for example, in the region (iii). In the case of the region (i) or (ii), however, the field components corresponding to $n_{r 1}$ vanish slowly with increasing $r$ in proportion to the Hankel functions, while the components corresponding to $n_{r 2}$ vanish very rapidly with increasing $r$.

\section{Electromagnetic Fields Near the Wire}

If the conditions

$$
\left|k_{r 1} r\right| \ll 1, \quad\left|k_{r 2} r\right| \ll 1
$$

are assumed in the vicinity of an extremely thin wire, electromagnetic fields near to the wire can be approximated by

$$
\begin{aligned}
E_{r} & \simeq E_{0} \frac{j 2\left(n_{r 2}^{2}-n_{r 1}^{2}\right)}{\pi n_{r 1}\left(\kappa_{1}-n_{z}^{2}-n_{r 1}^{2}\right)} \cdot \frac{e^{\mp j k} Z}{k_{0} r}, \\
E_{\phi} & \simeq 0, \\
E_{z} & \simeq-E_{0} \frac{2 n_{r 1} n_{z}}{\pi\left(\kappa_{3}-n_{r 1}^{2}\right)}\left\{\ln \frac{n_{r 1}}{n_{r 2}}\right\}\left\{1-\frac{\ln k_{r 2} r}{\ln k_{r 2} a}\right\} e^{\mp j k z^{Z}}, \\
H_{r} & \simeq 0,
\end{aligned}
$$




$$
\begin{gathered}
H_{\phi} \simeq E_{0} \frac{j 2 n_{z} \kappa_{3}}{\pi\left(\kappa_{3}-n_{r 1}^{2}\right)} \sqrt{\frac{\epsilon_{0}}{\mu_{0}}} \cdot \frac{n_{r 2}^{2}-n_{r 1}^{2}}{n_{r 1} n_{r 2}^{2}} \cdot \frac{e^{\mp j k} Z}{k_{0} r} \\
H_{z} \simeq E_{0} \frac{j 2 \kappa_{2}}{\pi n_{r 1}\left(\kappa_{1}-n_{z}^{2}-n_{r 1}^{2}\right)} \sqrt{\frac{\epsilon_{0}}{\mu_{0}}}\left\{\left(n_{r 1}^{2}-n_{r 2}^{2}\right) \ln \frac{j \gamma k_{0} r}{2}\right. \\
\left.+n_{r 1}^{2} \ln n_{r 1}-n_{r 2}^{2} \ln n_{r 2}\right\} e^{\mp j k_{z} Z}
\end{gathered}
$$

where

$$
\gamma=1.78107 \ldots \ldots
$$

Introducing the expression of $H_{\phi}$ into (15), we obtain

$$
I(z) \simeq E_{0} \frac{j 4 n_{z} \kappa_{3}\left(n_{r 2}^{2}-n_{r 1}^{2}\right)}{k_{0} n_{r 1} n_{r 2}^{2}\left(\kappa_{3}-n_{r 1}^{2}\right)} \sqrt{\frac{\epsilon_{0}}{\mu_{0}}} e^{\mp j k_{z} Z} .
$$

As the major component of the electric field around the wire is the $r$-component, the relation between $E_{r}(r, \phi, Z)$ and $I(Z)$ might be of interest. For this reason the ratio of $E_{r}$ to $I(Z)$ is calculated from (24) and (26). Introduction of (17) into the expression of the ratio finally leads to

$$
\frac{E_{r}(r, \phi, Z)}{I(z)} \simeq \frac{1}{2 \pi r n_{z}} \sqrt{\frac{\mu_{0}}{\epsilon_{0}}}
$$

It should be noticed here that this relation is just the same as in the case of an isotropic medium with the relative propagation constant of $n_{z}$.

\section{Conclusions}

Electromagnetic waves along an infinitely long conducting wire in a plasma medium with an axial static magnetic field have been analyzed theoretically. The detailed discussion was confined to the case of an extremely thin wire. Dependence of the relative propagation constants on the plasma and the cyclotron frequencies and their physical significance are explained in section 4. The behavior of the electromagnetic fields around the wire is discussed in section 5 . Although this theory involves approximations and assumptions, some information concerning the characteristics of linear antennas in a magneto-ionic medium can be derived from the results of this study. For example, the wavelength for the first approximation of the current distribution along an antenna wire placed in such a medium may be determined by (22).

The author acknowledges S. Adachi for his helpful discussions and K. Kato for his assistance in the computation.

\section{Appendix}

Derivation of (17). Taking the first terms of the expansions of the Hankel functions in (12) under the conditions $\left|k_{r 1} a\right|<<1$ and $\left|k_{r 2} a\right|<<1$ we obtain

$$
\frac{n_{r 1}^{2}\left(\kappa_{1}-n_{z}^{2}-n_{r 1}^{2}\right)\left(\kappa_{3}-n_{r 2}^{2}\right)}{n_{r 2}^{2}\left(\kappa_{1}-n_{z}^{2}-n_{r 2}^{2}\right)\left(\kappa_{3}-n_{r 1}^{2}\right)} \simeq \frac{\ln \left(j \gamma k_{r 2} a / 2\right)}{\ln \left(j \gamma k_{r 1} a / 2\right)} \simeq 1-\frac{\ln \left(n_{r 1} / n_{r 2}\right)}{\ln \left(j \gamma k_{r 1} a / 2\right)} \simeq 1-\frac{\ln \left(n_{r 1} / n_{r 2}\right)}{\ln k_{r 1} a} .
$$

Further approximation with the condition $\left|\ln \left(n_{r 1} / n_{r 2}\right)\right|<<\left|\ln k_{r 1} a\right|$ leads to (17) in the text. 


\section{References}

Ament, W. S., J. C. Katzin, M. Katzin, and B. Y.-C. Koo (1964), Impedance of a cylindrical dipole having a sinusoidal current distribution in a homogeneous anisotropic ionosphere, Radio Sci. J. Res. NBS/USNC-URSI 68D, No. 4, $379-405$.

Kogelnik, H. (1960), On electromagnetic radiation in magnetoionic media, J. Res. NBS 64D (Radio Prop.) No. 5, 515-523.

Marini, J. W. (1963), Radiation in a lossless magnetoionic medium at frequencies high relative to the electron gyrofrequency, J. Res. NBS 67D (Radio Prop.), No. 6, 707-716.

Mushiake, Y. (1964), Some characteristics of antennas in a magnetoionic medium, Summaries of papers of ICMCI Tokyo (1964), Part 1 (Microwaves) 381-382, Sept. 1964.

Wu, C. P. (1963), Radiation from dipoles in a magnetoionic medium, IEEE Trans. Ant. Prop. AP-1 1, 681-689.

(Paper 69D4-486) 